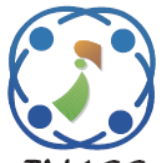

\title{
Multilevel Thresholding for Medical Image Segmentation Using Teaching- Learning Based Optimization Algorithm
}

\author{
Rajaraman Kalyani ${ }^{1 *}$ \\ Palani Duraisamy Sathya ${ }^{1}$ \\ Vadugapalayam Ponnuvel Sakthivel ${ }^{2}$ \\ ${ }^{1}$ Department of Electronics and Communication Engineering, Annamalai University, Chidambaram, India \\ ${ }^{2}$ Department of Electrical and Electronics Engineering, Government College of Engineering, Dharmapuri, India \\ * Corresponding author's Email: harebalu@gmail.com
}

\begin{abstract}
Medical image segmentation is the basic pre-processing step to infer information from the input image with RGB color space. In this paper, multilevel thresholding (MLT) with most optimistic objective functions such as Kapur and Otsu are used for image segmentation. But the MLT suffers from high execution time with the increase in number of threshold levels while exploring for optimal threshold. This difficulty is eased by the robust teachinglearning based optimization (TLBO) algorithm. It mimics the classroom environment where the student gains knowledge from the teacher. The main aspect of the TLBO is the use of less algorithm specific parameters in search process and it avoids premature convergence and getting trapped with sub-optimal solutions. The performance of TLBO algorithm is compared with cuckoo search (CS) algorithm at 4, 5, 6 and 7 threshold levels. Experimental results confirm that the Otsu based MLT outperform the Kapur objective function. Exploration and exploitation reveal the fast convergence and are confirmed by metrics such as computational time, peak signal to noise ratio (PSNR) and structural similarity index (SSIM). This affirms the inclusion of TLBO algorithm for precise medical image segmentation.
\end{abstract}

Keywords: Kapur, Otsu, Multilevel thresholding, Teaching-learning algorithm, Cuckoo search algorithm.

\section{Introduction}

Accurate diagnosis of eye diseases is still a difficult task and time consuming. Chorioretinitis, optic neuritis, age-related macular degeneration (AMD), diabetic retinopathy are some of the major causes of blindness worldwide, causing severe impact on individual's life [1]. Failure in drainage of aqueous fluid gives rise to glaucoma [2]. This results in gradual loss of vision due to increase in intra-ocular pressure. The symptoms of glaucoma and other causes of visual loss are similar, thus making early detection of glaucoma difficult. Another type of multifactorial eye disease called Dry eye syndrome (DE) directly has impact on the eye surface resulting in reduced vision.

Discrimination between melanoma and nonmelanoma is especially important as melanoma skin disease has a poor prognosis [3].
Melanoma skin cancer accounts for $75 \%$ of skin cancer deaths [4]. Early diagnosis through the preprocessing image segmentation step assists dermatologists with better visibility in clinical examination and for the visual inspection of skin lesion [5]. The most commonly occurring malignant tumor is Lung cancer. Small cell lung cancer (SCLC) and non-small cell lung cancer (NSCLC) types are associated with poor prognosis. These types of cancers at the initial stage spread locally and at advanced stage metastasise to lymph nodes and to other parts of the body [6].

Image segmentation is a pre- processing essential procedure used to analyse, extract meaningful information from the object of interest and it divides the images based on intensity, color and texture [7]. Generally, image segmentation techniques are categorized into thresholding-based, edge detection, region based and clustering methods. 
Edge detection method finds the object boundaries with the help of edge filters, whereas region-based method determines regions directly by spatial clustering (merging and splitting). Clustering techniques by fuzzy c-means, genetic algorithms are also used in image segmentation [8]. Wherein, thresholding is the histogram based, most simple, intuitive technique used to find the valleys between the peaks by optimal threshold values.

Generally, bilevel thresholding (BLT) is employed to segment the image into two classes. BLT can be extended as multilevel thresholding (MLT) for analysing complex images with more than two classes. Normally, thresholding technique can be categorized as global and local thresholding. Global thresholding selects only single threshold relying heavily on illumination. But local thresholding selects multiple thresholds by operating in smaller regions. Local thresholding is harder to implement [9]. Thus, the probability density of histogram is selected by parametric or nonparametric approaches irrespective of global (or) local category. As parametric approaches depend on initial conditions resulting in expensive computation, non-parametric approaches such as Otsu, Metaheuristic provide optimal solution to solve complex problems by combining rules and randomness [10].

Many algorithms such as Genetic algorithm (GA), Simulated annealing (SA), Ant colony optimization (ACO), Artificial bee colony (ABC), Differential evolution (DE), Differential search (DS) are available in literature [11]. The general draw backs of these algorithms are getting stuck with suboptimal point, poor segmentation in assessing complex images when there is an increase in threshold level, difficulty in fine tuning the control parameters. In the above-mentioned algorithms various parameters need to be controlled. So, it is important to pick appropriate parameters to get optimal values. But teaching-learning based optimization is the simple, and most efficient. It needs only common parameters such as population size and the number of iterations. It implements the approach of teacher imparting knowledge to the student. The highly experienced teacher always produces better results (grades) by training the students. No need of tuning any specific parameter to search the optimal threshold in this algorithm. In this paper, TLBO is proposed to solve the multilevel thresholding for image segmentation. The proposed paper considers the Kapur and Otsu as objective functions. This method is tested on three medical color images with 4, 5, 6 and 7 threshold levels and the efficacy of TLBO is compared with Cuckoo search (CS) algorithm.

The main aspects of TLBO algorithm is listed as three-fold:

- TLBO algorithm is easy and specific due to less use of algorithm specific parameters.

- Balance between exploration and exploitation is achieved and hence it results in lower number of iterations.

- Efficient and effective strategies of students learning from the teacher and through mutual interaction resulted in precise image segmentation.

The rest of this paper is organized as follows: Section 2 discusses the problem estimation of multilevel thresholding (MLT) methods using Kapur and Otsu. Section 3 introduces the overview of TLBO algorithm and its pseudocode for color medical mage segmentation. Section 4 describes the implementation of TLBO with MLT for color image segmentation. Section 5 presents the discussion of experimental results and finally concluding remarks are in Section 6.

\section{Problem estimation of multilevel thresholding (MLT)}

To segment the image more precisely, forecasting of proper threshold value is an essential task in simple, direct, accurate and robust thresholding technique and it is analysed by extracting histogram content from the input image [12].

\subsection{Multilevel thresholding}

Color images are distinguished into foreground and background by using more than two optimal thresholds (tri or quad levels) to segment the three components of $R, G, B$ offering excellent specificity [13]. Multiple threshold points classify the image into different classes giving the choice to analyse the target area.

$$
\begin{aligned}
& O_{1}(x, y)=\left\{i(x, y) \epsilon I \mid 0 \leq I(x, y) \leq m_{1}-1\right\} \\
& O_{2}(x, y)=\left\{i(x, y) \epsilon I \mid m_{1} \leq I(x, y) \leq m_{2}-1\right\} \\
& O_{i}(x, y)=\left\{i(x, y) \epsilon I \mid m_{i} \leq I(x, y) \leq m_{i+1}-\right. \\
& 1\} \ldots \\
& O_{r}(x, y)=\left\{i(x, y) \epsilon I \mid m_{r} \leq I(x, y) \leq L-1\right.
\end{aligned}
$$

where $t_{1}, t_{2}, t_{3}, t_{4} \ldots t_{i}, \ldots t_{r}$ indicate different thresholds. 
Different pixel-gray clusters are assigned based on the intensity value and each cluster will have the pixel value within specified range. In this proposed paper, the area to be examined is interpreted by segmenting the color images using maximising objective functions such as Kapur and Otsu.

\subsection{Kapur method (Maximum entropy)}

Entropy is used to predict the intended information from an image by estimating the uncertainty correlating the inter-pixel relations resulting in positive probabilities [14].

Let an image consists of $G$ maximum intensity gray level and their ranges from $\{0,1,2, \ldots,(G-1)\}$

$$
P_{i}=Q(i){ }_{N}, \quad(0 \leq i \leq(G-1))
$$

where $i$ ranges from 0 to 255 and $Q(i)$ indicates the number of pixels for the gray level $G$ and $N$ represents the total number of pixels in an image.

$$
N=\sum_{i=0}^{G-1} Q(i)
$$

Maximizing the objective function by:

$$
\begin{aligned}
& f(t)=F_{0}+F_{1} \\
& F_{0}=-\sum_{i=0}^{t-1} \frac{P_{i}}{X_{0}} \ln \frac{P_{i}}{X_{0}} ; \quad X_{0}=\sum_{i=0}^{t-1} P_{i} \\
& F_{1}=-\sum_{i=t}^{G-1} \frac{P_{i}}{X_{1}} \ln \frac{P_{i}}{X_{1}} ; \quad X_{1}=\sum_{i=t}^{G-1} P_{i}
\end{aligned}
$$

Thus, Kapur's entropy achieves unification of the histogram for image segmentation. Extension of Kapur's concept for Multilevel thresholding:

For $k$ optimal thresholds of an image $\left[t_{1}, t_{2}, \ldots, t_{k}\right]$ to maximise the objective function.

$$
f\left(\left[t_{1}, t_{2}, \ldots, t_{k}\right]\right)=F_{0}+F_{1}+\ldots+F_{k}
$$

where

$$
\begin{aligned}
& F_{0}=-\sum_{i=0}^{t_{1}-1} \frac{P_{i}}{X_{0}} \ln \frac{P_{i}}{X_{0}} ; \quad X_{0}=\sum_{i=0}^{t_{1}-1} P_{i} \\
& F_{1}=-\sum_{i=t_{1}}^{t_{2}-1} \frac{P_{i}}{X_{1}} \ln \frac{P_{i}}{X_{1}} ; \quad X_{1}=\sum_{i=t_{1}}^{t_{2}-1} P_{i} \\
& F_{2}=-\sum_{i=t_{2}}^{t_{3}-1} \frac{P_{i}}{X_{2}} \ln \frac{P_{i}}{X_{2}} ; X_{2}=\sum_{i=t_{2}}^{t_{3}-1} P_{i}, \ldots \\
& F_{k}=-\sum_{i=t_{k}}^{G-1} \frac{P_{i}}{X_{k}} \ln \frac{P_{i}}{X_{k}} ; \quad X_{K}=\sum_{i=t_{k}}^{G-1} P_{i}
\end{aligned}
$$

\subsection{Otsu method (between-class variance)}

Otsu's criteria predict the optimal threshold by maximising between-class variance to distinguish the focal point and the background [15].

Let an image with $G$ gray levels is divided into two classes namely $A_{0}$ and $A_{l}$ by a threshold level ' $t$ '

$A_{0}$ indicates gray levels from 0 to $t-1$ and $A_{1}$ from $t$ to $G-1$.

$$
\begin{aligned}
& A_{0}=P_{0} / x_{0}, \ldots P_{t-1} / x_{0} \\
& A_{1}=P_{t} / x_{1}, \ldots P_{G-1} / x_{1}
\end{aligned}
$$

where

$$
x_{0}=\sum_{i=0}^{t-1} P_{i} \quad \text { and } \quad x_{1}=\sum_{i=t}^{G-1} P_{i}
$$

Mean values $\mu_{0}$ and $\mu_{1}$ :

$$
\mu_{0}=\sum_{i=0}^{t-1} \frac{i \times P_{i}}{x_{0}} \text { and } \mu_{1}=\sum_{i=t}^{G-1} \frac{i \times P_{i}}{x_{1}}
$$

$\mu_{T}$ be the mean intensity of whole image

$$
\mu_{\mathrm{T}}=x_{0} \quad \mu_{0}+x_{1} \quad \mu_{1} \text { and } x_{0}+x_{1}=1
$$

According to Otsu's between-class variance discriminant analysis:

$$
\begin{aligned}
& y(t)=\sigma_{0}+\sigma_{1} \\
& \sigma_{0}=x_{0}\left(\mu_{0}-\mu_{T}\right)^{2} \\
& \sigma_{1}=x_{1}\left(\mu_{1}-\mu_{T}\right)^{2}
\end{aligned}
$$

Otsu's bilevel optimal threshold ' $t$ "' as

$$
t^{*}=\arg \max \{y(t)\} \quad 0 \leq t \leq G-1
$$

Extension of Otsu's concept for multilevel thresholding:

Original image is divided into $\mathrm{k}$ classes by ' $\mathrm{k}$ ' thresholds as $A_{0}$ ranges from $\left[0, \ldots, t_{1}-1\right], A_{1}$ as from $\left[t_{1}, \ldots, t_{2}\right.$ and $A_{k}$ as from $\left[t_{k}, \ldots, G-1\right]$, the optimal thresholds $\left(t_{0}{ }^{*}, t_{1}{ }^{*}, \ldots, t_{k}{ }^{*}\right)$ are selected by maximising $\mathrm{y}(\mathrm{t})$ as:

$$
\begin{array}{r}
\left(t_{0}{ }^{*}, t_{1}{ }^{*}, \ldots, t_{k}{ }^{*}\right)=\arg \max \{y(t)\} \quad 0 \leq t_{1} \leq \\
\ldots \leq t_{k} \leq G-1
\end{array}
$$

$$
\text { where } y(t)=\sigma_{0}+\sigma_{1}+\sigma_{2}+\cdots+\sigma_{k}
$$




$$
\begin{aligned}
\sigma_{0} & =x_{0}\left(\mu_{0}-\mu_{T}\right)^{2}, \\
\sigma_{1} & =x_{1}\left(\mu_{1}-\mu_{T}\right)^{2}, \ldots . \\
\sigma_{k} & =x_{k}\left(\mu_{k}-\mu_{T}\right)^{2} .
\end{aligned}
$$

With the increase in number of thresholds, computational time increases which limits the multilevel thresholding applications. This problem is overcome by predicting the perfect parameters of Kapur and Otsu multilevel thresholding using TLBO algorithm for excellent image segmentation. The proposed method maximises the Kapur's and Otsu's fitness function.

\section{Teaching- learning based optimisation (TLBO) algorithm}

Generally, the population-based algorithm is categorized as evolutionary based genetic algorithm (GA), differential evolution (DE), bacterial foraging (BF) and swarm intelligence based (PSO, artificial bee colony (ABC) etc. These probabilistic algorithms need to control only common parameters such as population size and number of iterations. That it is needless to control any specific parameters such as inertial weight, social variables etc is the main advantage of TLBO. So, chance for improper tuning is nullified and hence, the improved accuracy.

This novel population based TLBO algorithm proposed by R.V. Rao et al. (2011) [16-18] mimics the learning in classroom. Here, every student gains knowledge and puts effort to understand from another student to upgrade his/her cognizance. This algorithm operates in two modes namely, teacher and student mode.

\subsection{Teacher mode}

In this mode, the teacher transfers his/her knowledge to the student and attempt to enhance the mean result by improving the student's intelligence. The superior student in the whole population is believed as the teacher, as the teacher is the most experienced individual. Let $x$ be the number of subjects (design parameters) with $y$ number of students with population size $(P=1,2,3, \ldots, y)$, $M_{v, u}$ is the mean result of certain subject ' $\mathrm{v}$ ' as $(v=$ $1,2,3, \ldots, x)$.

The student gains knowledge from the teacher based on his/her capability as:

$$
D M_{v, u}=r_{u}\left(S_{v, p_{\text {best }, u}}-T_{f} M_{v, u}\right)
$$

$D M_{v, u}$ is the difference between the student and mean result of the students in every subject, $r_{u}$ is the range $[0,1], T_{f}$ is the constant, it may be 1 or 2 and selected randomly as: $T_{f}=\operatorname{round}[1+$ $\operatorname{rand}(0,1)\{2-1\}], S_{v, p_{\text {best } u}}$ is the best result of the student in subject v. $r_{u}$ and $T_{f}$ are developed arbitrarily and will not be submitted as input. Thus, this algorithm avoids tuning of $r_{u}, T_{f}$. Whereas, in PSO, GA algorithms required tuning of inertia weight, crossover\& mutation parameters.

Current solution in teacher phase is updated as:

$$
S_{v, p, u}^{\prime}=S_{v, p, u}+D M_{v, u}
$$

Updated value of $L_{v, p, u}$ is $L_{v, p, u}^{\prime}$. The best objective value from $L_{v, p, u}^{\prime}$ is accepted. The accepted values are fed as input to the learner mode.

\subsection{Student mode}

Learning is gained by communicating among them in this mode. The student learns from another student who is more knowledgeable than himself. Random selection of any two students, A, B, such that $S^{\prime}{ }_{\text {total }-A, u} \neq S_{\text {total-B,u }}^{\prime}$.

At the end of teacher mode, $S_{\text {total-A,u }}^{\prime}$ and $S_{\text {total-B,u }}^{\prime}$ are the updated results of $S_{\text {total-A,u }}$ and $S_{\text {total }-B, u}$.

$$
\begin{aligned}
& S^{\prime \prime}{ }_{v, A, u}=S_{v, A, u}^{\prime}+r_{u}\left(S_{v, A, u}^{\prime}-S_{v, B, u}^{\prime}\right) \\
& \text { if } S_{\text {total-A,u}}^{\prime}>S_{\text {total-B,u }}^{\prime} \\
& S^{\prime \prime}{ }_{v, A, u}=S^{\prime}{ }_{v, A, u}+r_{u}\left(S^{\prime}{ }_{v, B, u}-S_{v, A, u}^{\prime}\right) \\
& \text { if } S_{\text {total-B,u}}^{\prime}>S_{\text {total }-A, u}^{\prime}
\end{aligned}
$$

Thus, $S^{\prime \prime}{ }_{v, A, u}$ is accepted if it provides the optimal fitness output.

\section{Algorithmic search tracks for optimal threshold}

Self-organisation of the system in each iteration is by set of rules to attain optimal solutions through search steps namely:

Learning process 1: Initialization of parameters: Initialise the population size (class of students), number of iterations and the (different subjects) design variables. This step generates the initial random values (threshold values) within upper and lower limits of threshold value (color threshold value for each component) [0-255]. 


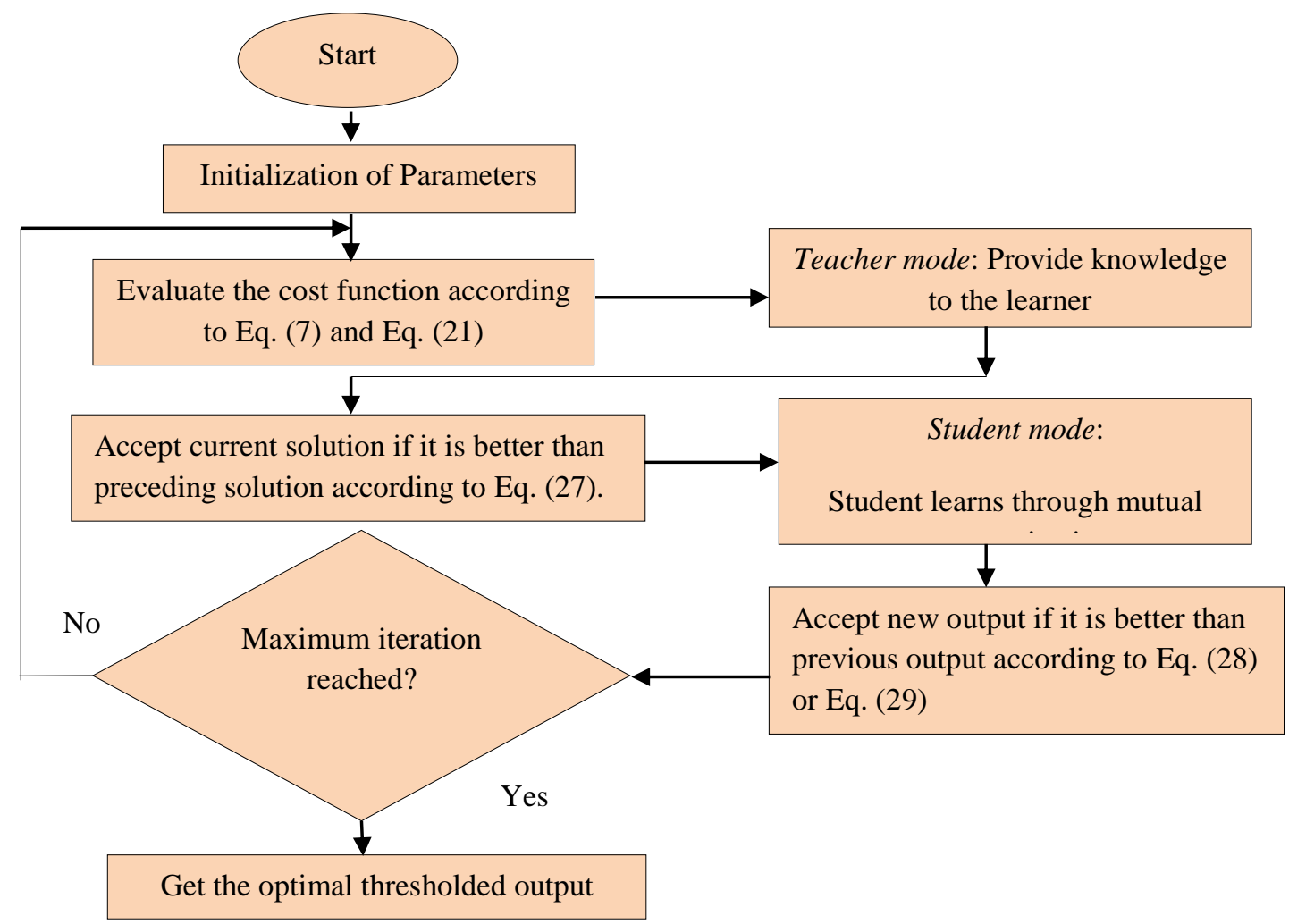

Figure. 1 Flow chart of teaching-learning based optimisation for multilevel thresholding-based image segmentation

Learning process 2: Generation of population: Initialise randomly the population (students), design variables (different subjects).

Learning process 3: $\quad$ Evaluate the cost function: Fitness function (results of the student) is evaluated according to Eq. (7) and Eq. (21).

Learning process 4: $\quad$ Teacher mode: Students learn from teacher (best fitness solution) as per Eq. (27).

Learning process 5: $\quad$ Student mode: Students learn within themselves through mutual interactions as per Eq. (28) or Eq. (29). The parameter value obtained in a subject depicts the knowledge possessed by the learner.

Learning process 6: Stop Condition: The optimal threshold is obtained when maximum iteration is reached or-else the search process is repeated from learning process.

The flowchart for Teaching-learning based multilevel thresholding is given in Fig. 1 and this algorithm requires only common control parameters such as population size 50 with 100 iterations.

\section{Experimental results and discussions}

Proficiency of various bio-inspired algorithms to target the global threshold for image segmentation is presented in the literature. The tactical approach of teaching-learning idea is implemented in the proposed paper for precise medical image segmentation as the image segmentation is the basic pre-processing step for efficacious analysis. The most propitious objective functions such as Kapur and Otsu are utilized in this paper for effective diagnosis to aid various field medical experts. The extraction of global threshold is accomplished by TLBO based MLT, implemented in Matlab 7.0, Processed in Intel core 2 Duo Processor $(3 \mathrm{GHz}), 2$ GB RAM. The superior performance of TLBO based MLT technique is compared with Cuckoo search algorithm by testing on medical color images. Section 5.1 presents the specifications and simulation details for benchmark images. Section 5.2 deals with the solution excellence through metrices such as CPU time, PSNR (Peak Signal to Noise Ratio), SSIM (Structural Similarity Index).

\subsection{Specifications and simulation details for medical images}

Eminent performance of TLBO based multilevel thresholding for medical imaging segmentation aided with Kapur and Otsu objective functions are compared with recent metaheuristic cuckoo search algorithm. Illustration to exhibit precise segmentation is depicted through the input Fig. 2 


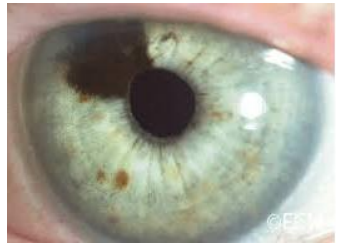

(a)

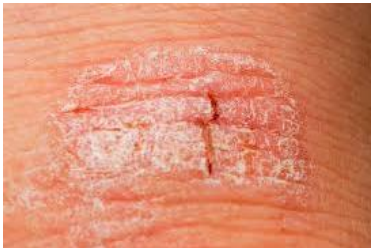

(b)

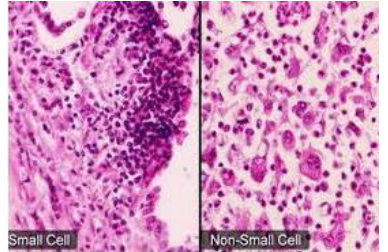

(c)

Figure.2 Input images: (a) iris and (b) psoriasis and (c) cancer cell

Table 1. Optimal threshold and objective values of each algorithm under Kapur's method

\begin{tabular}{|c|c|c|c|c|c|}
\hline Iris & $\begin{array}{l}\text { No. of } \\
\text { thresholds }\end{array}$ & Red Band & Green Band & Blue Band & $\begin{array}{l}\text { Objective } \\
\text { values }\end{array}$ \\
\hline \multirow{4}{*}{ TLBO } & 4 & 97143193210 & 63148184225 & 5594144196 & 54.898357 \\
\hline & 5 & 86137183201238 & 88121135186227 & 100157186206236 & 62.161371 \\
\hline & 6 & $\begin{array}{c}100130145186210 \\
231\end{array}$ & 6881135149202241 & $\begin{array}{c}93135165196208 \\
225\end{array}$ & 68.960123 \\
\hline & 7 & $\begin{array}{c}3389124151170201 \\
229\end{array}$ & $\begin{array}{c}5282120169200217 \\
231\end{array}$ & $\begin{array}{c}59115132167186 \\
199230\end{array}$ & 76.29345 \\
\hline \multirow{4}{*}{ CS } & 4 & 93134160194 & 4483170219 & 81130166191 & 54.953195 \\
\hline & 5 & 96152168217231 & 85150198220238 & 57108139177218 & 61.864947 \\
\hline & 6 & $\begin{array}{c}59108137172179 \\
201\end{array}$ & $\begin{array}{c}56130166184216 \\
226\end{array}$ & $\begin{array}{c}89126167199210 \\
238\end{array}$ & 68.548185 \\
\hline & 7 & $\begin{array}{c}52107131154177 \\
191241 \\
\end{array}$ & $\begin{array}{c}497299117134167 \\
223\end{array}$ & $\begin{array}{c}6187118169187218 \\
235 \\
\end{array}$ & 76.545304 \\
\hline Psoriasis & $\begin{array}{l}\text { No. of } \\
\text { thresholds }\end{array}$ & Red Band & Green Band & Blue Band & $\begin{array}{c}\text { Objective } \\
\text { values }\end{array}$ \\
\hline \multirow{4}{*}{ TLBO } & 4 & 115149182189 & 68109146180 & 59113140187 & 48.192272 \\
\hline & 5 & 117145151176216 & 60144155178199 & 34100164175198 & 55.084957 \\
\hline & 6 & $\begin{array}{c}65138158192217 \\
231 \\
\end{array}$ & 5873130183195211 & $\begin{array}{c}53124162174200 \\
206 \\
\end{array}$ & 60.393391 \\
\hline & 7 & $\begin{array}{c}44118134162197 \\
218226\end{array}$ & $\begin{array}{c}70117137178186 \\
204220\end{array}$ & $\begin{array}{c}69105141179197 \\
205215\end{array}$ & 65.500999 \\
\hline \multirow{4}{*}{ CS } & 4 & 118139162190 & 5168127184 & 6385169175 & 47.860342 \\
\hline & 5 & 80143168209229 & 74107120167203 & 80100128183205 & 53.810493 \\
\hline & 6 & $\begin{array}{c}115129149181201 \\
249\end{array}$ & 5884109143178199 & $\begin{array}{c}89124150162188 \\
200\end{array}$ & 61.439928 \\
\hline & 7 & $\begin{array}{c}63119136159177 \\
207228 \\
\end{array}$ & $\begin{array}{c}4163127141190199 \\
227\end{array}$ & $\begin{array}{c}3587149154162196 \\
212 \\
\end{array}$ & 65.241273 \\
\hline $\begin{array}{c}\text { Cancer } \\
\text { Cell }\end{array}$ & $\begin{array}{c}\text { No. of } \\
\text { thresholds }\end{array}$ & Red Band & Green Band & Blue Band & $\begin{array}{c}\text { Objective } \\
\text { values }\end{array}$ \\
\hline \multirow{4}{*}{ TLBO } & 4 & 5186153212 & 6499160200 & 4996158220 & 56.919151 \\
\hline & 5 & 4394162210237 & 105129174200215 & 4576150182221 & 63.532267 \\
\hline & 6 & 4297119136178233 & $\begin{array}{c}105130160175200 \\
215\end{array}$ & 5397130166184216 & 71.079891 \\
\hline & 7 & $\begin{array}{c}72110123140174 \\
214234 \\
\end{array}$ & $\begin{array}{c}3963103161193213 \\
236 \\
\end{array}$ & $\begin{array}{c}4786107135170217 \\
233 \\
\end{array}$ & 78.724537 \\
\hline \multirow{4}{*}{ CS } & 4 & 56114164225 & 85140166238 & 61107145212 & 56.09857 \\
\hline & 5 & 3767130180223 & 3974159174200 & 82154180201212 & 63.207386 \\
\hline & 6 & 5281119132170202 & $\begin{array}{c}54127150180215 \\
244 \\
\end{array}$ & 4085167181207231 & 70.832186 \\
\hline & 7 & $\begin{array}{c}6698131151180213 \\
237\end{array}$ & $\begin{array}{c}3892137150187208 \\
235 \\
\end{array}$ & $\begin{array}{c}6489133150188212 \\
239 \\
\end{array}$ & 78.28899 \\
\hline
\end{tabular}


Received: July 10, 2020. Revised: September 6, 2020.

Table 2. Optimal threshold and objective values of each algorithm under Otsu's method

\begin{tabular}{|c|c|c|c|c|c|}
\hline Iris & $\begin{array}{l}\text { No. of } \\
\text { thresholds }\end{array}$ & Red Band & Green Band & Blue Band & $\begin{array}{l}\text { Objective } \\
\text { values }\end{array}$ \\
\hline \multirow{4}{*}{ TLBO } & 4 & 105137174208 & 84105155199 & 64134173210 & 12831.45103 \\
\hline & 5 & 98143160172212 & 56137176190216 & 70108132181226 & 12872.43467 \\
\hline & 6 & $\begin{array}{c}6587130167212 \\
225\end{array}$ & $\begin{array}{c}86120173184212 \\
247\end{array}$ & $\begin{array}{c}73114149181201 \\
231\end{array}$ & 12902.38326 \\
\hline & 7 & $\begin{array}{c}62104148160194 \\
214220\end{array}$ & $\begin{array}{c}61119145179192 \\
210244\end{array}$ & $\begin{array}{c}70104130142170 \\
201220 \\
\end{array}$ & 12933.74747 \\
\hline \multirow{4}{*}{$\mathrm{CS}$} & 4 & 71138176190 & 86140183198 & 66118152203 & 12830.10077 \\
\hline & 5 & 94138154200234 & 93142182199233 & 5297125175193 & 12863.67986 \\
\hline & 6 & $\begin{array}{c}81124159166212 \\
253\end{array}$ & $\begin{array}{c}45101144177207 \\
228\end{array}$ & $\begin{array}{c}54121137155171 \\
204\end{array}$ & 12899.1428 \\
\hline & 7 & $\begin{array}{c}667694134169196 \\
225 \\
\end{array}$ & $\begin{array}{c}70107120166190 \\
206232 \\
\end{array}$ & $\begin{array}{c}6897127150160177 \\
208 \\
\end{array}$ & 12935.59554 \\
\hline Psoriasis & $\begin{array}{l}\text { No. of } \\
\text { thresholds }\end{array}$ & Red Band & Green Band & Blue Band & $\begin{array}{l}\text { Objective } \\
\text { values }\end{array}$ \\
\hline \multirow{4}{*}{ TLBO } & 4 & 34147186212 & 115149179185 & 5890130174 & 12932.56548 \\
\hline & 5 & 36145170191221 & 118157164174188 & 6781119158235 & 12945.34907 \\
\hline & 6 & $\begin{array}{c}100147165171209 \\
214\end{array}$ & $\begin{array}{c}95123137147166 \\
200\end{array}$ & 4987110123150159 & 12967.14158 \\
\hline & 7 & $\begin{array}{c}5082149158173 \\
203233 \\
\end{array}$ & $\begin{array}{c}68116118135138 \\
153184 \\
\end{array}$ & $\begin{array}{c}7093114131158173 \\
245\end{array}$ & 12977.84313 \\
\hline \multirow{4}{*}{$\mathrm{CS}$} & 4 & 7073192229 & 116143175181 & 87121150197 & 12927.76393 \\
\hline & 5 & 83156188212233 & 116121134144188 & 73117156218242 & 12940.54683 \\
\hline & 6 & $\begin{array}{c}74128156184194 \\
214\end{array}$ & $\begin{array}{c}105130154173191 \\
228\end{array}$ & 3386113136163186 & 12963.39826 \\
\hline & 7 & $\begin{array}{c}6573141168190 \\
202224 \\
\end{array}$ & $\begin{array}{c}67109128153162 \\
187242 \\
\end{array}$ & $\begin{array}{c}76117136161193 \\
200233 \\
\end{array}$ & 12969.88247 \\
\hline $\begin{array}{c}\text { Cancer } \\
\text { Cell }\end{array}$ & $\begin{array}{c}\text { No. of } \\
\text { thresholds }\end{array}$ & Red Band & Green Band & Blue Band & $\begin{array}{c}\text { Objective } \\
\text { values }\end{array}$ \\
\hline \multirow{4}{*}{ TLBO } & 4 & 66149191243 & 46113148195 & 37130176212 & 17325.84439 \\
\hline & 5 & 79149171217245 & 4594132174207 & 92114167176225 & 17370.12923 \\
\hline & 6 & $\begin{array}{c}110151187194229 \\
241\end{array}$ & 4956101151192226 & $\begin{array}{c}109157178199226 \\
251\end{array}$ & 17374.98726 \\
\hline & 7 & $\begin{array}{c}78104120152195 \\
214234 \\
\end{array}$ & $\begin{array}{c}3985127167193220 \\
234 \\
\end{array}$ & $\begin{array}{c}74106132155185 \\
204217 \\
\end{array}$ & 17427.83353 \\
\hline \multirow{4}{*}{ CS } & 4 & 116150197236 & 76131180193 & 73117170223 & 17316.44073 \\
\hline & 5 & 60136183206231 & 63105107144192 & 95139170216235 & 17367.45992 \\
\hline & 6 & $\begin{array}{c}116154173205229 \\
246 \\
\end{array}$ & 4473101171199217 & $\begin{array}{c}32110160197226 \\
242 \\
\end{array}$ & 17369.94509 \\
\hline & 7 & $\begin{array}{c}79116145162190 \\
206235 \\
\end{array}$ & $\begin{array}{c}4677101144184211 \\
216 \\
\end{array}$ & $\begin{array}{c}6380128163197 \\
217220 \\
\end{array}$ & 17424.62311 \\
\hline
\end{tabular}

such as Iris $(143 \times 197 \times 512)$, Psoriasis $(147 \times 223) \times$ $512)$ and Cancer cell $(151 \times 223) \times 321)$ medical color images.

\subsection{Result excellence validation through metrics}

Generally, practical problems in segmenting medical images decide the number of threshold levels. 


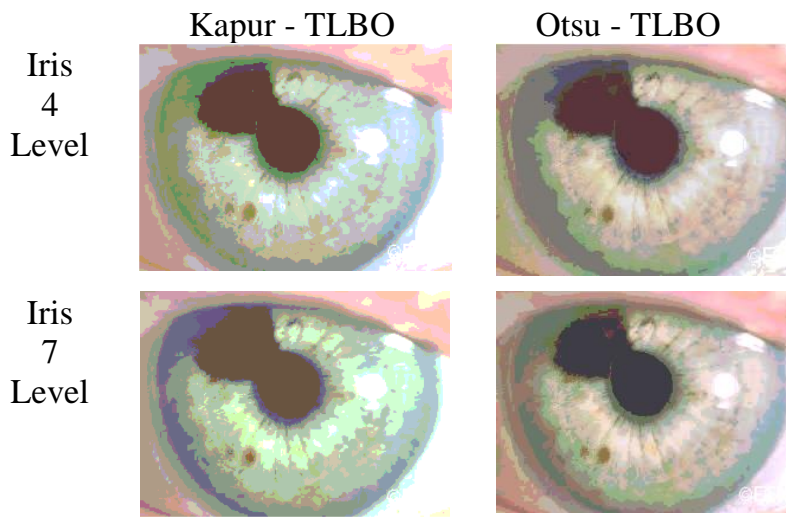

(a)

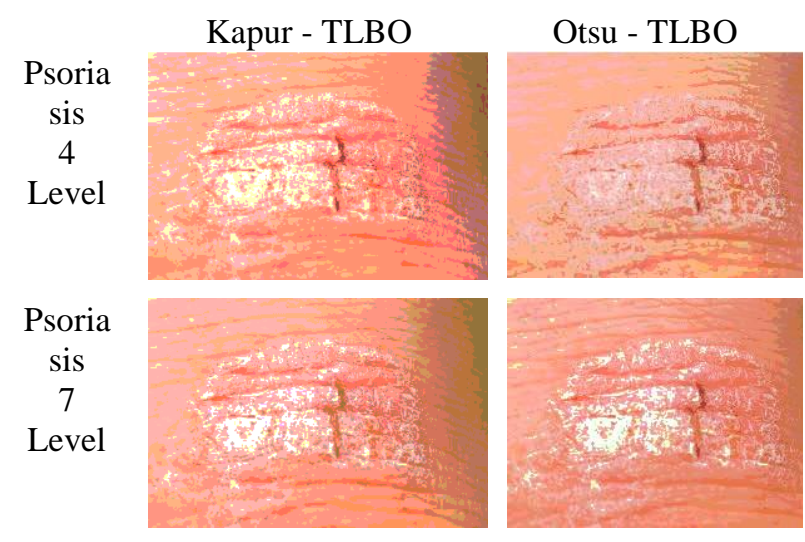

(b)

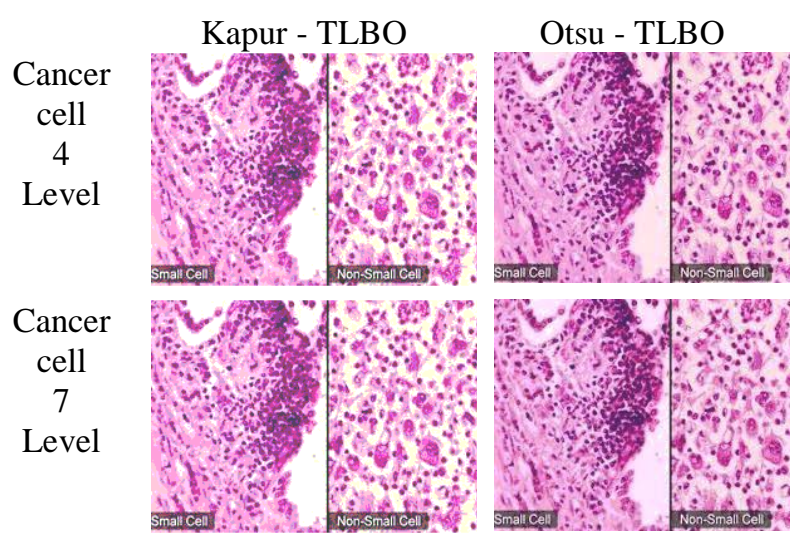

(c)

Figure. 3 Segmentation results of TLBO: (a) iris -4 and 7 th level of Kapur and Otsu method, (b) psoriasis -4 and 7 th level of Kapur and Otsu method, and (c) cancer cell 4 and 7 th level of Kapur and Otsu method

Qualitative analysis is carried out at 4, 5, 6 and 7 threshold levels for achieving the intensified and diversified dimensional search to maximise the most promising Kapur and Otsu's fitness function.

\subsubsection{Objective function analysis}

The perfect threshold values with red band, green band, blue band by searching in each component of medical color images and the corresponding objective function values for Kapur, and Otsu are listed in the Tables 1-2. The visual representations are in Fig. 3. The best objective functions of TLBO based MLT are obtained comparing with Cuckoo Search (CS) algorithm. TLBO is the simple and straightforward technique as the students learn directly from the teacher. The mutual interaction of the learner mode achieves the local search and hence the minimum number of iterations is required for TLBO algorithm. The teacher and the student modes drive the search for optimal threshold efficiently and effectively without any deviation. But the CS algorithm resulted in expensive computation to attain the stable arbitrary parameters to model levy flight in meeting the time constraint [19]. Thus, TLBO aided with Kapur and Otsu aid in precise image segmentation avoiding the delay with minimum number of iterations and by stable intensified, diversified search.

For example, from the Table 2, the Psoriasis objective values prove the accurate image segmentation of TLBO based Otsu's method with the values $12932.56548,12945.34907,12967.14158$ and 12977.84313 at 4,5,6 and 7 threshold levels respectively, compared to CS based Otsu with 12927.76393, 12940.54683, 12963.39826 and 12969.88247 on $4,5,6$ and 7 threshold levels respectively. The reason behind TLBO achieving the best objective values is the students gaining knowledge from the expert teacher and through mutual interaction, exploiting the unknown points to check the feasibility of unknown area.

\subsubsection{Computational time}

Analysis of CPU metric plays a major role since less computational time is required to meet the real time demands. and Tables 3-4 show that the optimal output is reached with fewer iterations indicating less time to converge. Low run time during the threshold levels 4, 5, 6 and 7 shows the possibility of inclusion of this algorithm for real time applications. Usually, computational time increases with the increase in threshold levels. But the TLBO based MLT achieves the computation within a reasonable amount of time.

Hence TLBO based Otsu, despite increase in number of thresholds, is able to obtain much more detailed segmented information in most of the cases compared to TLBO based Kapur. 
Table 3. CPU time (s) of Kapur method

\begin{tabular}{|c|c|c|c|}
\hline $\begin{array}{c}\text { Input } \\
\text { Images }\end{array}$ & $\begin{array}{c}\text { No. of } \\
\text { thresholds }\end{array}$ & TLBO & CS \\
\hline \multirow{4}{*}{ Iris } & 4 & 1.215792 & 1.224314 \\
\cline { 2 - 4 } & 5 & 1.358120 & 1.358151 \\
\cline { 2 - 4 } & 6 & 1.712741 & 1.711436 \\
\cline { 2 - 4 } & 7 & 2.115270 & 2.126810 \\
\hline \multirow{4}{*}{ Psoriasis } & 4 & 1.013081 & 1.020765 \\
\cline { 2 - 4 } & 5 & 1.342133 & 1.343780 \\
\cline { 2 - 4 } & 6 & 1.790157 & 1.795964 \\
\hline \multirow{3}{*}{\begin{tabular}{c} 
Cell \\
\cline { 2 - 4 }
\end{tabular}} & 7 & 2.184970 & 2.171153 \\
\cline { 2 - 4 } & 4 & 1.283570 & 1.312118 \\
\cline { 2 - 4 } & 6 & 1.274780 & 1.345613 \\
\hline \multirow{4}{*}{} & 7 & 2.291484 & 2.298572 \\
\hline
\end{tabular}

Table 4. CPU time (s) of Otsu method

\begin{tabular}{|c|c|c|c|}
\hline $\begin{array}{c}\text { Input } \\
\text { Images }\end{array}$ & $\begin{array}{c}\text { No. of } \\
\text { thresholds }\end{array}$ & TLBO & CS \\
\hline \multirow{4}{*}{ Iris } & 4 & 0.812147 & 0.82587 \\
\cline { 2 - 4 } & 5 & 1.311921 & 1.3241 \\
\cline { 2 - 4 } & 6 & 1.671549 & 1.673846 \\
\cline { 2 - 4 } & 7 & 2.291764 & 2.314642 \\
\hline \multirow{4}{*}{ Psoriasis } & 4 & 0.978412 & 0.991055 \\
\cline { 2 - 4 } & 5 & 1.383759 & 1.385748 \\
\cline { 2 - 4 } & 6 & 1.748942 & 1.749102 \\
\hline \multirow{3}{*}{\begin{tabular}{c} 
Cell \\
\cline { 2 - 4 }
\end{tabular}} & 7 & 2.258431 & 2.279461 \\
\cline { 2 - 4 } & 5 & 0.978411 & 0.993518 \\
\cline { 2 - 4 } & 6 & 0.995741 & 1.10541 \\
\hline \multirow{4}{*}{} & 7 & 2.188576 & 2.274615 \\
\hline
\end{tabular}

\subsubsection{Peak signal to noise ratio (PSNR)}

Peak signal to noise ratio indicates the segmentation quality of an image in direct proportion as it depends on intensity values of an image.

Visual depiction of segmented image from original image confirms the ability and accuracy of
TLBO based MLT with high PSNR and low RMSE. PSNR is stated as:

$$
P S N R=20 \times \log _{10}\left(\frac{255}{R M S E}\right)
$$

where $M S E$ refers the root mean-squared error, $I$ and $I^{\wedge}$ refers the original and thresholded images and $\mathrm{M} \times \mathrm{N}$ indicates the dimensions of an image.

Tables 5 shows the comparison of TLBO based MLT with CS in terms of PSNR to specify the accuracy of an image for the Kapur and Otsu based fitness function. Thus, from the Table 5 and 6 , Otsu fitness function take upper hand than Kapur fitness function indicating the object of interest to be infered from the segmented image even with increase in number of thresholds. Proper threshold value is determined by PSNR thereby avoiding over and under segmentation. Experimental results reveal that the results are in favour of TLBO based MLT. High PSNR of TLBO with low MSE affirms the low degree of distorted image.

\subsubsection{Structural similarity index (SSIM)}

Structural similarity index measures the consistency between the true and segmented image. Higher value of SSIM confirms the quality of original image. SSIM is given as:

$$
\operatorname{SSIM}(\mathrm{x}, \mathrm{y})=\frac{\left(2 \mu_{t} \mu_{s}+c_{1}\right)\left(2 \sigma_{t s}+c_{2}\right)}{\left(\mu_{t}^{2}+\mu_{s}^{2}+c_{1}\right)\left(\sigma_{t}^{2}+\sigma_{s}^{2}+c_{2}\right)}
$$

where $\mu_{t}$ and $\mu_{s}$ are mean intensity of true and segmented image, $\sigma_{t}$ and $\sigma_{s}$ are the standard deviation of true and segmented image, $\sigma_{t s}$ is covariance of true and segmented image and $c_{1}, c_{2}$ are constants.

Table 5. MSE and PSNR of Kapur's and Otsu's methods

\begin{tabular}{|c|c|c|c|c|c|c|c|c|c|}
\hline \multirow{3}{*}{$\begin{array}{c}\text { Input } \\
\text { Images }\end{array}$} & \multirow{2}{*}{$\begin{array}{c}\text { Threshold } \\
\text { Levels }\end{array}$} & \multicolumn{4}{|c|}{ Kapur's method } & \multicolumn{4}{c|}{ Otsu's method } \\
\cline { 3 - 10 } & & TLBO & \multicolumn{2}{|c|}{ CS } & \multicolumn{2}{|c|}{ TLBO } & \multicolumn{3}{c|}{ CS } \\
\cline { 3 - 10 } & MSE & PSNR & MSE & PSNR & MSE & PSNR & MSE & PSNR \\
\hline \multirow{3}{*}{ Iris } & 4-level & 315.12 & 23.15 & 294.86 & 23.28 & 286.19 & 23.56 & 331.56 & 22.93 \\
\cline { 2 - 11 } & 7-level & 161.80 & 26.04 & 226.43 & 24.58 & 146.40 & 26.48 & 139.28 & 26.69 \\
\hline \multirow{3}{*}{\begin{tabular}{c} 
Psoriasis \\
\cline { 3 - 10 }
\end{tabular}} & 4-level & 307.17 & 23.26 & 456.04 & 21.54 & 282.86 & 23.62 & 286.53 & 23.56 \\
\cline { 2 - 10 } & 7-level & 130.18 & 26.99 & 162.68 & 26.02 & 100.32 & 28.12 & 239.33 & 24.34 \\
\hline \multirow{2}{*}{$\begin{array}{c}\text { Cancer } \\
\text { Cell }\end{array}$} & 4-level & 349.53 & 22.70 & 386.01 & 22.26 & 290.13 & 23.50 & 289.51 & 23.51 \\
\cline { 2 - 10 } & 7-level & 159.05 & 26.12 & 117.86 & 27.42 & 119.39 & 27.36 & 113.86 & 27.57 \\
\hline
\end{tabular}


Table 6. SSIM of Kapur method

\begin{tabular}{|c|c|c|c|}
\hline $\begin{array}{c}\text { Input } \\
\text { Images }\end{array}$ & $\begin{array}{c}\text { Threshold } \\
\text { Levels }\end{array}$ & TLBO & CS \\
\hline \multirow{2}{*}{ Iris } & 4-level & 0.8668 & 0.8611 \\
\cline { 2 - 4 } & 7-level & 0.8832 & 0.8391 \\
\hline \multirow{2}{*}{ Psoriasis } & 4-level & 0.9753 & 0.9633 \\
\cline { 2 - 4 } & 7-level & 0.9875 & 0.9808 \\
\hline \multirow{2}{*}{$\begin{array}{c}\text { Cancer } \\
\text { Cell }\end{array}$} & 4-level & 0.9507 & 0.9457 \\
\cline { 2 - 4 } & 7-level & 0.9761 & 0.9719 \\
\hline
\end{tabular}

Table 7. SSIM of Otsu method

\begin{tabular}{|c|c|c|c|}
\hline $\begin{array}{c}\text { Input } \\
\text { Images }\end{array}$ & $\begin{array}{c}\text { Threshold } \\
\text { Levels }\end{array}$ & TLBO & CS \\
\hline \multirow{2}{*}{ Iris } & 4-level & 0.8624 & 0.8713 \\
\cline { 2 - 4 } & 7-level & 0.8779 & 0.89 \\
\hline \multirow{2}{*}{ Psoriasis } & 4-level & 0.9667 & 0.9637 \\
\cline { 2 - 4 } & 7-level & 0.9901 & 0.9681 \\
\hline \multirow{2}{*}{$\begin{array}{c}\text { Cancer } \\
\text { Cell }\end{array}$} & 4-level & 0.957 & 0.9554 \\
\cline { 2 - 4 } & 7-level & 0.9772 & 0.9748 \\
\hline
\end{tabular}

Thus, the Tables 7-8 infer the superior performance of TLBO with maximum value of SSIM for Kapur and Otsu objective function.

\section{Conclusion}

To obtain the desired details from the image, the simple, population-based, teaching-learning based optimization algorithm with multilevel thresholding segmentation technique is used. The non-parametric fitness functions such as Kapur and Otsu are very flexible and computationally effective. They aid to exploit the global point through optimal prediction of threshold values. The searching tactics are implemented with the set of students (learners) gaining knowledge from the trained, experienced (best solution) teacher. The two states namely, teacher and student state enhance the class result. Thus, the experimental results show the outstanding achievement of Otsu based TLBO than Kapur based TLBO. CPU time, PSNR and SSIM authenticate the outperformance of TLBO based MLT compared with CS by testing on medical color images.

\section{Conflicts of Interest}

The authors declare no conflict of interest.

\section{Author Contributions}

The contributions of authors are as follows: conceptualization: Palani Duraisamy Sathya; methodology, software, validation, formal analysis, investigation, and resources: Rajaraman Kalyani,
Palani Duraisamy Sathya, and Vadugapalayam Ponnuvel Sakthivel; original draft preparation, review and editing, visualization, supervision, and project administration: Palani Duraisamy Sathya; and funding acquisition, Rajaraman Kalyani.

\section{Acknowledgments}

Acknowledgments are to show that the article is supported by what organization. For example, "This work was supported by the National Nature Science Foundation under Grant No. 405”.

\section{References}

[1] J. Orlosky, Y. Itoh, M. Ranchet, K. Kiyokawa, J. Morgan and H. Devos, "Emulation of Physician Tasks in Eye-Tracked Virtual Reality for Remote Diagnosis of Neurodegenerative Disease", IEEE Transactions on Visualization and Computer Graphics, Vol.23, No. 4, pp.1302-1311, 2017.

[2] E. Ouabida, A. Essadike, and A. Bouzid, "Automated segmentation of ophthalmological images by an optical based approach for early detection of eye tumor growing", Physica Medica, Vol. 48, pp.37-46, 2018.

[3] Z. Yu, X. Jiang, F. Zhou, J. Qin, D. Ni and S. Chen and T. Wang, "Melanoma Recognition in Dermoscopy Images via Aggregated Deep Convolutional Features", IEEE Transactions on Biomedical Engineering, Vol.66, No.4, pp.1006-1016, 2018.

[4] F. Yu, M. Zheng, A. Zhang and Z. Han, "A cerium oxide loaded glycol chitosan nanosystem for the treatment of dry eye disease", Journal of Controlled Release, Vol.315, pp.4054, 2019.

[5] J. Hagerty, J. Stanley, H. Almubarak, N. Lama, R. Kasmi,, P. Guo and W.V. Stoecker, "Deep Learning and Handcrafted Method Fusion: Higher Diagnostic Accuracy for Melanoma Dermoscopy Images", IEEE Journal of Biomedical and Health Informatics, Vol.26, No. 4,pp.1385-1391,2019.

[6] W. Chen, H. Wei, S. Peng, J. Sun, X. Qiao and B.Liu, "HSN: Hybrid Segmentation Network for Small Cell Lung Cancer Segmentation", IEEE Access, Vol.7, pp.75591-75603,2019.

[7] T. Sağ, and M. Çunkaş, "Color image segmentation based on multiobjective artificial bee colony optimization", Applied Soft Computing, Vol.34, pp.389-401, 2015.

[8] R. M. Haralick, and L.G. Shapiro, "Image segmentation techniques", Computer Vision, 
Graphics, and Image Processing, Vol.29, No.1, pp.100-132, 1985.

[9] U. Mlakar, B. Potočnik and J. Brest, "A hybrid differential evolution for optimal multilevel image thresholding", Expert Systems with Applications, Vol.65, pp. 221-232,2016.

[10] X. Zhao, M. Turk, W. Li, K. Lien and G. Wang, "A multilevel image thresholding segmentation algorithm based on two-dimensional $\mathrm{K}-\mathrm{L}$ divergence and modified particle swarm optimization", Applied Soft Computing, Vol.48, pp.151-159,2016.

[11] G. Sun, A. Zhang, Y. Yao and Z. Wang, "A novel hybrid algorithm of gravitational search algorithm with genetic algorithm for multi-level thresholding", Applied Soft Computing, Vol.46, pp.703-730, 2016.

[12] P. D. Sathya and R. Kayalvizhi, "Comparison of intelligent techniques for multilevel thresholding problem", In: Proc. Of International journal of signal and imaging systems engineering, pp.4357, 2012.

[13] M. Abd Elaziz, S. Bhattacharyya and S. Lu, "Swarm Selection Method for Multilevel Thresholding Image Segmentation", Expert Systems with Applications, Vol.138, pp.112818, 2019.

[14] S. Sarkar and S. Das, "Multilevel Image Thresholding Based on 2D Histogram and Maximum Tsallis Entropy- A Differential Evolution Approach", IEEE Transactions on image processing, Vol.22, No.12, pp. 4788-4797, 2013.
[15] T. Sağ, and M. Çunkaş, "Color image segmentation based on multiobjective artificial bee colony optimization", Applied Soft Computing, Vol. 34, pp. 389-401, 2015.

[16] R. V. Rao, V.J. Savsani and D.P. Vakharia, "Teaching-learning-based optimization: A novel method for constrained mechanical design optimization problems", Computer-Aided Design, Vol.43, No. 3, pp.303-315, 2011.

[17] H. Singh Gill, B. Singh Khehra, A. Singh and L. Kaur, "Teaching-learning-based optimization algorithm to minimize cross entropy for Selecting multilevel threshold value", Egyptian Informatics Journal, Vol.20, No.1, pp.11-25, 2019.

[18] R. V. Rao, V.J. Savsani and D.P. Vakharia, "Teaching-Learning-Based Optimization: An optimization method for continuous non-linear large-scale problems", Information Sciences, Vol.183, No.1, pp.1-15, 2012.

[19] S. Pare, A. Kumar, V. Bajaj, G.K. Singh, "An efficient method for Multilevel Colour image thresholding using cuckoo search algorithm based on minimum cross entropy", Applied Soft Computing, Vol.61, pp. 570-592, 2017. 